\title{
Optimization of Tapered Photonic Crystal Fibers for Blue-Enhanced Supercontinuum Generation
}

Møller, Uffe; Sørensen, Simon Toft; Larsen, Casper; Jakobsen, Christian ; Johansen, Jeppe; Moselund, Peter M.; Thomsen, Carsten L.; Bang, Ole

\section{Published in:}

Proceedings of Nonlinear Photonics

\section{Publication date:}

2012

Document Version

Publisher's PDF, also known as Version of record

Link back to DTU Orbit

Citation (APA):

Møller, U., Sørensen, S. T., Larsen, C., Jakobsen, C., Johansen, J., Moselund, P. M., Thomsen, C. L., \& Bang, O. (2012). Optimization of Tapered Photonic Crystal Fibers for Blue-Enhanced Supercontinuum Generation. In Proceedings of Nonlinear Photonics (pp. JW4D.1). Optical Society of America.

\section{General rights}

Copyright and moral rights for the publications made accessible in the public portal are retained by the authors and/or other copyright owners and it is a condition of accessing publications that users recognise and abide by the legal requirements associated with these rights.

- Users may download and print one copy of any publication from the public portal for the purpose of private study or research.

- You may not further distribute the material or use it for any profit-making activity or commercial gain

- You may freely distribute the URL identifying the publication in the public portal 


\title{
Optimization of Tapered Photonic Crystal Fibers for Blue-Enhanced Supercontinuum Generation
}

\author{
Uffe Møller, ${ }^{1, *}$ Simon T. Sørensen, ${ }^{1}$ Casper Larsen, ${ }^{1}$ Christian Jakobsen ${ }^{2}$, Jeppe \\ Johansen $^{2}$, Peter M. Moselund ${ }^{2}$, Carsten L. Thomsen ${ }^{2}$, and Ole Bang ${ }^{1,2}$ \\ ${ }^{1}$ DTU Fotonik - Dept. of Photonics Engineering, Technical University of Denmark, DK-2800 Kgs. Lyngby, Denmark \\ ${ }^{2}$ NKT Photonics A/S, Blokken 84, DK-3400 Birkerød, Denmark \\ *ufmo@fotonik.dtu.dk
}

\begin{abstract}
Tapering of photonic crystal fibers is an effective way of shifting the dispersive wavelength edge of a supercontinuum spectrum down in the deep-blue. We discuss the optimum taper profile for blue-enhanced supercontinuum generation.
\end{abstract}

(C) 2012 Optical Society of America

OCIS codes: (060.4370) Nonlinear Optics, fibers; (320.6629) Supercontinuum generation.

\section{Introduction}

Supercontinuum (SC) sources generating light in the visible part of the electromagnetic spectrum have proven to be valuable tools in various biological applications [1]. For these applications it is beneficial that as much light as possible is available in the visible part of the spectrum. In the recent years, much research has been devoted to tailoring the photonic crystal fibers (PCFs) for optimizing the desired SC spectrum. Tapering of fibers have shown to be an effective way to blueshift the short wavelength edge of the $\mathrm{SC}$ by means of changing the fiber dispersion and increasing the nonlinearity $[2,3]$. In this work, we present an asymmetrical PCF taper fabricated directly on the draw-tower. The fiber is tapered back to its initial diameter to facilitate better handling and coupling efficiency, and to allow for an investigation of the asymmetry. We demonstrate high power SC generation with extended bandwidth and, by introducing the concept of a group-acceleration mismatch [4,5], we give a clear interpretation of the results and provide a recipe for optimizing the taper shape for maximum bandwidth and available power in the blue edge of the spectrum.

An SC spectrum largely consists of a solitonic red edge linked to a dispersive wave (DW) blue edge through a complex trapping mechanism. As the solitons are redshifting they cause the DWs to blueshift through group-velocity (GV) matching [6]. The position of the blue edge for a given fiber can hence be estimated from numerically calculated GV curves if the position of the solitonic edge is known. Figure 1(a) illustrates the blue edge wavelength as a function of hole-to-pitch ratio and pitch assuming group velocity matching to a loss edge of $2300 \mathrm{~nm}$ or $50 \mathrm{~nm}$ below the 2nd ZDW. The GV profile of the fiber, and hence the link between the spectral edges, can be altered by tapering $[2,4]$.
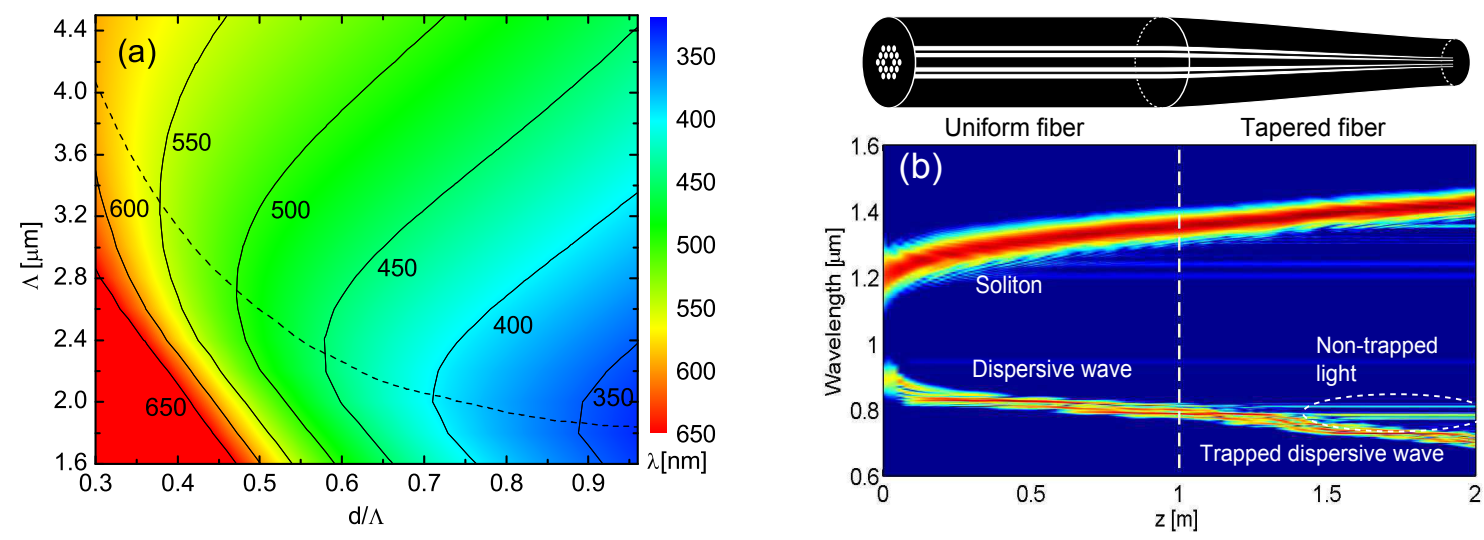

Fig. 1: (a) Blue edge wavelength $\lambda$ as a function of hole-to-pitch ratio $\mathrm{d} / \Lambda$ and pitch $\Lambda$ assuming group velocity matching to a loss edge of $2300 \mathrm{~nm}$ or $50 \mathrm{~nm}$ below the 2nd ZDW. The dashed line indicates the optimum $\Lambda$ for a given $\mathrm{d} / \Lambda$. (b) Spectral evolution of a $20 \mathrm{fs}$ fundamental soliton and trapped wave through a fiber taper with an initial $1 \mathrm{~m}$ uniform fiber. 
In a uniform fiber a soliton can trap and blueshift a DW as long as it keeps redshifting and decelerating. However, when a soliton and trapped DW enter a taper, the soliton undergoes a relatively larger change in GV (deceleration), which diminishes the trapping potential and causes light to escape. The trapping process and leakage is illustrated in Fig. 1(b) with a careful numerical simulation of the propagation of a soliton and seeded DW through a tapered fiber; the DW clearly starts shedding light in the tapered part of the fiber. We have recently demonstrated that the taper induced relative change in GV, or Group-Acceleration Mismatch (GAM), is responsible for the amount of blueshifted light; more light is blueshifted when the GAM is minimised by making the down-tapering more gradual. This can be understood as an enhancement of the interaction between the soliton and DW due to a minimization of the walk-off.

\section{Experiments}

By fabricating an asymmetric taper where the fiber is tapered back to its original diameter, the concept of GAM can be verified experimentally. The PCF taper was fabricated directly on the draw tower, which offers unprecedented control of the fiber parameters and excellent reproducibility. Figure 2(a) shows the spectra obtained when pumping the fiber with a high-power $1064 \mathrm{~nm}$ ps laser and compares the spectra obtained when pumping from the short and long downtapering side of the taper with that of a uniform fiber of a similar length. The taper had $3 \mathrm{~m}$ uniform fiber before and after the tapered section, and the dispersion of the uniform fiber and the taper waist is shown in Fig. 2(b). Tapering yields a clear extension of the spectral bandwidth, and, as expected due to the lower GAM, pumping from the long down-tapering side significantly enhances the available power in the extended bandwidth as illustrated in Fig. 2(c).
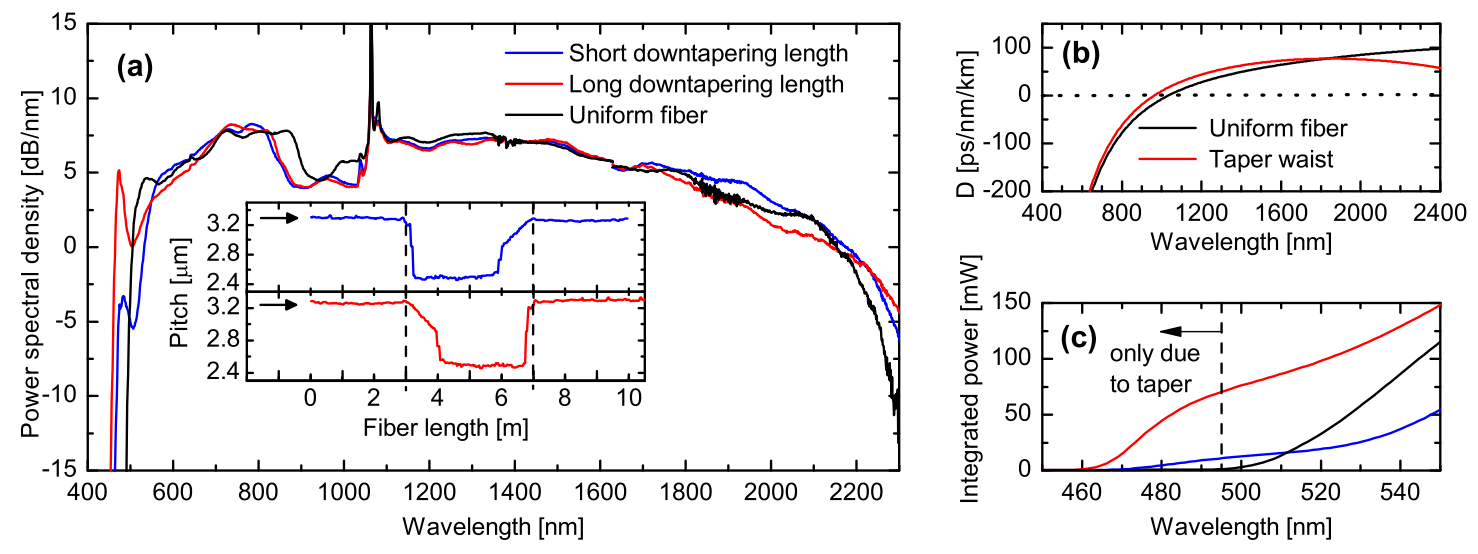

Fig. 2: (a) Experimental output spectra when pumping the asymmetric taper from the short (blue line) and long (red line) downtapering ends. The spectrum of a $10 \mathrm{~m}$ uniform fiber (black line) is shown for comparison. The inset shows the tapering profile and the arrows indicate the pump direction. (b) Calculated dispersion of the uniform fiber (black line) and the taper waist (red line). (c) Integrated power in the blue edge for the fibers shown in (a). The vertical line indicate the blue edge of the uniform fiber $(495 \mathrm{~nm})$, all power shifted below this wavelength is generated in the taper.

\section{Conclusion}

In conclusion, we have numerically and experimentally verified the importance of the concept of group-acceleration matching (GAM) to find the optimal taper shape. It was demonstrated that the taper degree determines the blue edge and the taper shape has a strong impact on the power in the blue edge. A longer down-tapered section yields a higher power in the blue edge of the spectrum due to a correspondingly lower GAM.

\section{References}

1. N. Savage, "Supercontinuum sources," Nat. Photonics 3, 114-115 (2009).

2. J. C. Travers, "Blue extension of optical fibre supercontinuum generation," J. Opt. 12, 113,001 (2010).

3. A. Kudlinski, A. K. George, J. C. Knight, J. C. Travers, A. B. Rulkov, S. V. Popov, and J. R. Taylor, "Zero-dispersion wavelength decreasing photonic crystal fibers for ultraviolet-extended supercontinuum generation," Opt. Express 14, 5715-5722 (2006).

4. S. T. Sørensen, A. Judge, C. L. Thomsen, and O. Bang, "Optimum fiber tapers for increasing the power in the blue edge of a supercontinuum-group-acceleration matching," Opt. Lett. 36, 816-818 (2011).

5. S. T. Sørensen, U. Møller, C. Larsen, P. M. Moselund, C. Jakobsen, J. Johansen, T. V. Andersen, C. L. Thomsen, and O. Bang, "Deep-blue supercontinuum sources with optimum taper profiles - verification of GAM," Opt. Express. 20, 10635-10645 (2012).

6. A. V. Gorbach and D. M. Skryabin, "Light trapping in gravity-like potentials and expansion of supercontinuum spectra in photonic-crystal fibres," Nat. Photonics 1, 653-657 (2007) 